\title{
Timing, LOINC Axis 3
}

National Cancer Institute

\section{Source}

National Cancer Institute. Timing, LOINC Axis 3. NCI Thesaurus. Code C40567.

The chronological relationship between when an observation is made and other relevant associated periods of time. For example, the timing axis specifies whether a given observation represents the value of a measurement at a moment (point) in time, an average of the measurement over a period of time ( 24 hours, 72 hours, a nursing shift, a clinical encounter), a minimum or maximum over a period of time (24 hour maximum), or its ordinal rank within a period of time (first, second, last). 